\title{
Outcome Analysis of Unstable Posterior Ring Injury of the Pelvis: Comparison between Percutaneous Iliosacral Screw Fixation and Conservative Treatment
}

\author{
Po-Han Chen, Wei-Hsiu Hsu, Yen-Yao Li, Tsan-Wen Huang, Tsung-Jen Huang, Kuo-Ti Peng
}

Background: Unstable posterior ring injuries of the pelvis are a challenge to pelvic surgeons, and their treatment has been controversial. The functional outcomes of such injuries following either percutaneous iliosacral screw fixation or conservative treatment remain to be elucidated.

Methods: We conducted a retrospective analysis of 32 consecutive patients with unstable pelvic ring injuries who were treated with percutaneous placement of iliosacral screws (group 1) or conservative means (group 2) from January 2002 to September 2009. Radiographic, clinical, and functional outcomes were compared between the two treatment groups.

Results: $\quad$ Patients who underwent percutaneous iliosacral screw fixation after pelvic trauma had better functional results than those treated conservatively, as per the Majeed grading system. In addition, patients in group 1 demonstrated better results for general health and mental health on the 36 item Short Form Health Status Survey. Patients in group 1 also demonstrated less residual displacement on radiography at 1 year follow up than those in group 2 . Finally, patients in group 1 had better pain relief at 1 month and 1 year follow ups than those in group 2.

Conclusions: Percutaneous iliosacral screw fixation for unstable posterior pelvic ring injuries results in less residual displacement at medium term follow up, and better pain relief at short and medium term follow up, than does conservative treatment. Better functional outcomes were observed at 1 year follow up as compared with conservative treatment.

(Biomed J 2013;36:289-294)

Key words: percutaneous iliosacral screws, pelvic ring injury, pelvis fracture, sacrum fracture sacroiliac joint disruption

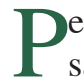
elvic injuries with posterior ring instability, including sacroiliac (SI) diastasis, fracture-dislocation of the SI joint, and sacral fractures, have been challenging to pelvic surgeons, and their treatment remains controversial. ${ }^{[1-3]}$ Several studies have indicated that conservative treatment

\begin{abstract}
At a Glance Commentary
Scientific background of the subject

Pelvis ring injuries without adequate treatment may lead to poor prognosis. Several treatment strategies have been proved effective. Placement of iliosacral screws was shown to provide excellent biomechanical stability and satisfactory results in outcome, and the percutaneous means have even less complications.
\end{abstract}

\section{What this study adds to the field}

This study provided a comparison of radiographic and functional outcomes of percutaneous iliosacral screw fixation and conservative treatment. Percutaneous placement of iliosacral screws can result in less residual displacement, better pain relief, and better functional outcomes. for these injuries may achieve acceptable results without disabling late sequelae. ${ }^{[1-3]}$ Other studies have indicated that patients with unstable posterior ring injury who are treated conservatively have poor long-term outcomes, including prolonged immobility, late back pain, and a high rate of

From the Department of Orthopedic Surgery, Chang Gung Memorial Hospital at Chiayi, Chang Gung University College of Medicine, Taoyuan, Taiwan

Received: Aug. 3, 2012; Accepted: Dec. 3, 2012

Correspondence to: Dr. Kuo-Ti Peng, Department of Orthopaedic Surgery, Chang Gung Memory Hospital at Chiayi. 6, W. Sec., Jiapu Rd., Puzi, Chiayi 613, Taiwan (R.O.C.). Tel: 886-5-3621000; ext. 2855; Fax: 886-5-3623001; E-mail: mr3497@cgmh.org.tw

DOI: $10.4103 / 2319-4170.112757$ 
nonunion or malunion..$^{[4-7]}$ It has been shown that surgical intervention, such as external fixation, internal fixation, or hybrid fixation, could provide adequate stability for pelvic fracture. ${ }^{[4-7]}$ Among those approaches, percutaneous placement of iliosacral screws, a minimally invasive approach, was shown to provide excellent biomechanical stability and satisfactory results in fracture reduction, early weight bearing, union rate, pain relief, and patient return to work..$^{[4,8,9]}$ Compare to open procedures, percutaneous iliosacral screwing also decreased the invasive nature and the incidence of wound infections. ${ }^{[10,11]}$ The stability of the SI joint results from a combination of bony structure and intrinsic and extrinsic ligaments. The SI joints provide shock absorption for the spine, along with torque conversion, allowing the transverse rotations that occur in the lower extremity to be transmitted up the spine. The SI joint also provides a locking mechanism that facilitates stability during the push-off phase of walking. ${ }^{[12]}$ Few studies have shed light on the functional outcomes of unstable posterior pelvic ring injury following either percutaneous iliosacral screw fixation or conservative treatment. Thus the present study was conducted to investigate the radiographic and functional results of posterior pelvic ring injury following percutaneous iliosacral screw fixation as compared with conservative treatment.

This investigation was approved by the Ethics Committee and Institutional Review Board of the Chang-Gung Memorial Hospital.

\section{METHODS}

The retrospective study involved consecutive patients who were treated at the authors' institution for unstable posterior pelvic ring injuries from January 2002 to September 2009. We searched International Classification of Diseases -9 (ICD-9) code 808 of hospital discharge and emergency department diagnoses in this period. The patients were brought to the emergency department because of falls or motor-vehicle accidents. To be eligible for inclusion in the study, patients had to be skeletally mature and to required evidence of traumatic unstable posterior pelvic ring injury on plain radiographs. This was defined as lateral compression (LC) type II or III injury, anteroposterior compression (APC) type II or III injury, vertical shear (VS) injury, or a combined-mechanism injury that was unstable as per the Young-Burgess classification system. ${ }^{[13]}$ Patients also required a completed 1 year of follow up to be included in the study. Patients with bilateral SI disruption, skeletal immaturity, systemic bone disease, neurological injuries of the lumbosacral plexus, urological injuries, and/or posterior osteosynthesis using means other than iliosacral screws were excluded.

There were 240 patients found with ICD- 9 code 808 treated in authors' institution within this period, 38 patients matched the inclusion criteria, and 32 patients completed the follow up. The remaining 6 patients were lost to follow up as they died cause of reasons unassociated with this injury. Group 1 consisted of 15 patients with a mean age of 45 years (range, 18-83 years) who were treated using percutaneous iliosacral screw fixation. Group 2 consisted of 17 patients with a mean age of 51 years (range, 18-72 years) who were treated by conservative means. The groups did not differ significantly in terms of gender, age, injury side, trauma mechanism, and classification [Table 1].

Before January 2005, in the authors' institution, all patients presenting with unstable pelvic ring injuries were treated nonsurgically or with posterior osteosynthesis, using means other than iliosacral screws. After January 2005, the author's institution started to treat unstable pelvic ring fracture by using percutaneous iliosacral screw for available of pelvic surgeon. The patients enrolled in the study were thus divided into two groups: Group 1 consisted of patients treated using fluoroscopy-assisted percutaneous iliosacral screw placement by a single orthopaedic surgeon (K-T P) after January 2005 [Figure 1], and group 2 consisted of patients treated nonsurgically without posterior construct for pelvic ring and January 2005. Patients in group 1, surgical management were performed after vital sign stable, usually 3 to 7 days after the day of injury. The purpose of surgical intervention was to achieve better anatomical position through fluoroscopically controlled closed reduction technique and percutaneous iliosacral screwing, and therefore enhancing the clinical results, such as pain control and functional improvement. In cases associated with anterior pelvic ring disruption, such as vertical shear and APC3 injuries, osteosynthesis with anterior plating was performed to improve stability.

Table 1: Demographic data and clinical characteristics

\begin{tabular}{lccc}
\hline & $\begin{array}{c}\text { Group 1 } \\
(n=15)\end{array}$ & $\begin{array}{c}\text { Group 2 } \\
(n=17)\end{array}$ & $p$ value \\
\hline Age & $45.13 \pm 23.17$ & $50.76 \pm 17.09$ & 0.523 \\
Gender & 7 & 5 & 0.474 \\
$\quad$ Male & 8 & 12 & \\
$\quad$ Female & & & \\
Injury side & 8 & 5 & 0.281 \\
$\quad$ Left & 7 & 12 & \\
$\quad$ Right & & & \\
Trauma mechanism & 3 & 1 & 0.322 \\
$\quad$ Fall & 12 & 16 & \\
$\quad$ MVA* & & & \\
Classification of Young-Burgess & 2 & 3 & 0.984 \\
LC*2 & 1 & 2 & \\
LC3 & 2 & 2 & \\
APC 2 & 2 & 2 & \\
APC3 & 8 & 8 & \\
VS* & & & \\
\hline
\end{tabular}

Abbreviations: MVA: Motor vehicle accident; LC: Lateral compression; APC: Anterior-posterior compression; VS: Vertical shear 


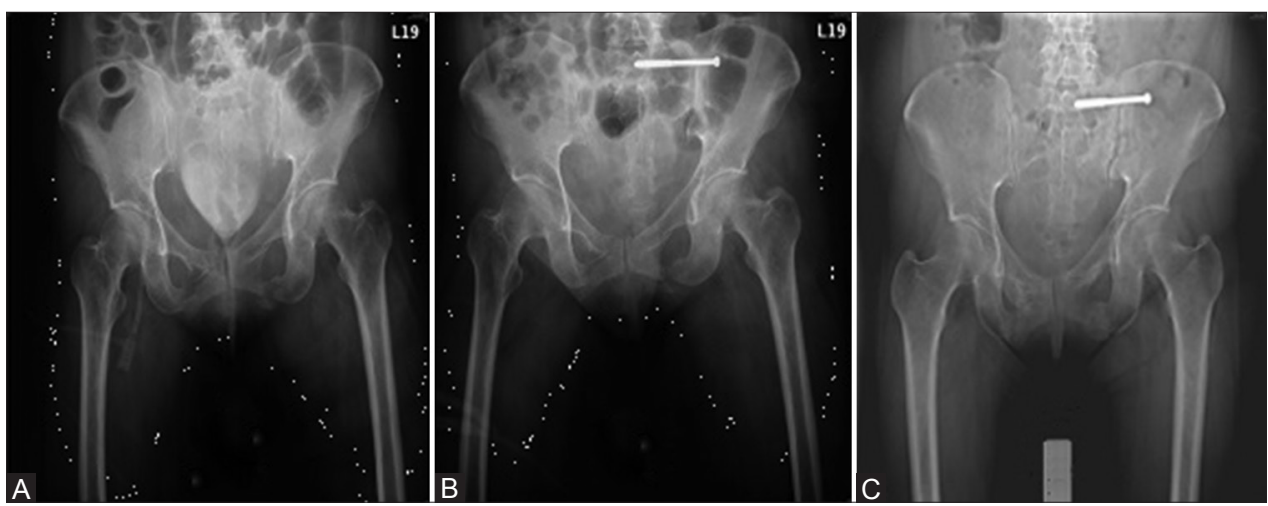

Figure 1: (A) Preoperative pelvic AP radiograph of a 42 y/o female patient suffered from LC3 type injury and left iliosacral joint dislocation. (B) Postoperative pelvic radiograph revealed anatomical reduction and adequate iliosacral screwing. (C) One year follow up pelvic radiograph showed bony union in anatomical position that patient did not suffer from back pain.

Serial radiographs were obtained at initial presentation and during 1-year follow up. The vertical proximal displacement of the hemi-pelvis was measured on standard AP radiographs as suggested by Henderson [Figure 2]. ${ }^{[3]}$ Initial, residual, and increased displacement after pelvic injury were recorded. The proportion of patients exhibiting residual displacement of more than $5 \mathrm{~mm}$ at 1-year follow up was compared between groups. Visual analogue scale (VAS) scores measuring pain at initial presentation and at 1-month and 1-year follow up were recorded and compared between groups. Improvement in the VAS score was defined as a decrease of the score relative to the initial VAS score at both 1-month and 1-year follow up. Subjective functional outcomes were assessed using the 36-item Short Form Health Status Survey (SF-36) and the Majeed grading system for functional results after pelvic trauma. The SF-36 is based on evaluation of eight health concepts and two component summaries: Physical functioning, role physical, bodily pain, general health, vitality, social functioning, role emotional, mental health, physical component summary, and mental component summary. A transformed scale score of 0 to 100 was calculated for each of these health concepts. Functional results graded using the Majeed system included assessment subscale scores for pain, work, sitting, sexual intercourse, and standing, with ratings of excellent, good, fair, or poor at 1-year follow up. ${ }^{[14]}$ PASW Statistics 18 software (Winwrap Basic copyright; SPSS Inc, Chicago, IL, USA) was used for statistical analysis. Outcomes for the two patient groups for displacement, VAS score, SF-36 score, and Majeed pelvis subscale score were compared using the Wilcoxon rank sum test; the proportions of patients with various Majeed pelvis grades and numbers of patients with more than $5 \mathrm{~mm}$ of displacement were compared using the Fisher's exact test. The level of statistical significance was set at 0.05 . Complications associated with sciatic never injury, dislocation or infection were also recorded.

Patients in group 1, non-weightbearing mobilization with 2 crutches was allowed but weight bearing was

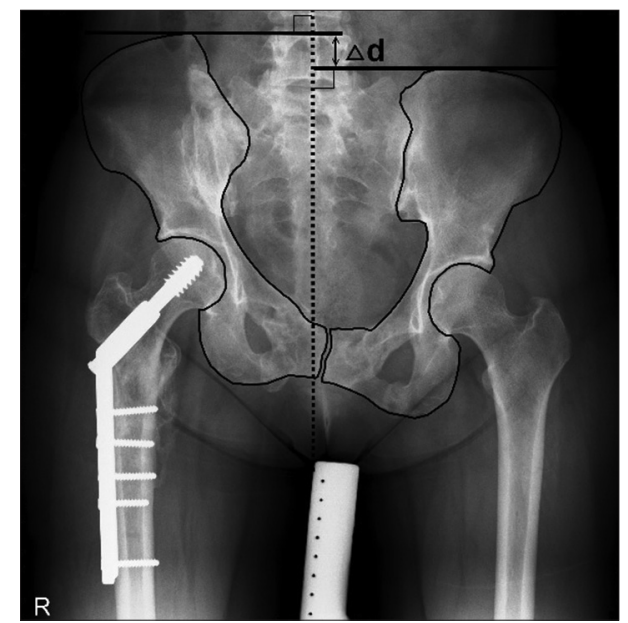

Figure 2: Measurement of proximal displacement. The vertical proximal displacement of the hemi-pelvis was measured on standard AP radiographs. $\Delta$ d: vertical distance of bilateral iliac crest on standard anterior-posterior radiography.

avoided for 6 weeks until radiographic evidence of SI joint restoration was confirmed. Physiotherapy to improve gait pattern was occasionally prescribed thereafter, for example, for hip extensor or abductor strengthening for 1.5 to 3 months. Patients in Group 2 were instructed to avoid weight bearing activity with skin traction for 3 months but passive assisted abduction with physiotherapist was permitted. Partial-weight bearing with 2 crutches was allowed 6 months later.

\section{RESULTS}

The mean amount of vertical proximal displacement of the hemi-pelvis at initial presentation for groups 1 and 2 were $4 \mathrm{~mm}$ and $4 \mathrm{~mm}$, respectively; the mean amounts at 1-year follow up were $3 \mathrm{~mm}$ and $6 \mathrm{~mm}$, respectively. Thus, at 1 year follow up, group 2 demonstrated additional displacement of $2 \mathrm{~mm}$, whereas group 1 showed a reduction in displacement of $1 \mathrm{~mm}$. This difference was statistically 
significant ( $p=0.024$ ) [Table 2]. In group 1, 2 of 15 patients had residual displacement of more than $5 \mathrm{~mm}$, compared with 11 of 17 patients in group $2(p=0.004)$ [Table 3]. Furthermore, in group 2, 2 of 17 patients had residual displacement of more than $10 \mathrm{~mm}$ (11 $\mathrm{mm}$ and $23 \mathrm{~mm})$, compared with none in group $1(p=0.001)$.

The mean \pm standard deviation (SD) VAS scores of group 1 at initial presentation, 1-month follow up, and 1-year follow up were $9.06 \pm 0.79,3.73 \pm 1.48$, and $2.73 \pm 1.43$, respectively; those of group 2 were $9.05 \pm 0.55,6.71 \pm 0.91$, and $4.17 \pm 0.81$, respectively. The differences in VAS scores between groups at 1 -month ( $p<0.001$, respectively) and 1 -year ( $p=0.001$, respectively) follow up were statistically significant, as were the differences in improvement in VAS scores between groups at 1 -month $(p<0.001$, respectively) and 1-year ( $p=0.001$, respectively) follow up [Table 4].

According to the SF-36 evaluation, group 1 had better results than group 2 for general health and mental health aspects at 1-year follow up ( $p=0.032$ and 0.043 , respectively) [Table 5]. In Majeed functional assessment, group 1 had better mean scores than group 2 for pain ( $25.00 \pm 7.32$ vs. $20.59 \pm 6.09 ; p=0.028)$; work ( $16.33 \pm 4.33$ vs. $10.57 \pm 4.60 ; p=0.006)$; and sitting $(8.00 \pm 1.85$ vs. $6.71 \pm 1.72 ; p=0.049)$. However, no significant differences were found between the two groups

Table 2: Comparison of proximal displacement

\begin{tabular}{lccc}
\hline & $\begin{array}{c}\text { Group 1 } \\
(n=15)\end{array}$ & $\begin{array}{c}\text { Group 2 } \\
(n=17)\end{array}$ & $p$ value \\
\cline { 2 - 3 } & \multicolumn{2}{c}{ Mean \pm SD $(\mathrm{mm})$} & \\
\hline Previous displacement & $4.13 \pm 2.33$ & $3.73 \pm 2.43$ & 0.532 \\
Residual displacement & $3.63 \pm 2.95$ & $6.34 \pm 5.25$ & $0.024^{*}$ \\
Progress displacement & $-0.51 \pm 2.71$ & $2.61 \pm 4.69$ & 0.613 \\
\hline
\end{tabular}

Abbreviation: SD: Standard deviation; ${ }^{*} p<0.05$

Table 3: Residual displacement

\begin{tabular}{lccc}
\hline & Group 1 $(n=15)$ & Group 2 $(n=17)$ & $p$ value \\
\hline Residual displacement & & & \\
$>5 \mathrm{~mm}$ & 2 & 11 & $0.004^{*}$ \\
$<5 \mathrm{~mm}$ & 13 & 6 & \\
\hline$* p 0.05$ & & &
\end{tabular}

Table 4: Results of visual analogue scale

\begin{tabular}{lccc}
\hline VAS & $\begin{array}{c}\text { Group 1 } \\
(n=15)\end{array}$ & $\begin{array}{c}\text { Group 2 } \\
(n=17)\end{array}$ & $p$ value \\
\cline { 2 - 3 } & \multicolumn{2}{c}{ Mean \pm SD } & \\
\hline Initial & $9.06 \pm 0.79$ & $9.05 \pm 0.55$ & 0.932 \\
1 month follow up & $3.73 \pm 1.48$ & $6.71 \pm 0.91$ & $<0.001 * * *$ \\
12 months follow up & $2.73 \pm 1.43$ & $4.17 \pm 0.81$ & $0.001 * *$ \\
Improvement after 1 month & $5.33 \pm 1.23$ & $2.35 \pm 1.11$ & $<0.001 * * *$ \\
Improvement after 12 months & $6.33 \pm 1.11$ & $4.88 \pm 1.05$ & $0.001 * *$ \\
\hline Abbreviations: VAS: Visual analogue scale; SD: Standard deviation; \\
$* * p<0.01 ; * * *$ value $<0.001$
\end{tabular}

Biomed J Vol. 36 No. 6

November - December 2013 for sexual intercourse (3.00 $\pm 1.00 \mathrm{vs.} 2.88 \pm 0.93 ; p=0.473)$ and standing $(26.27 \pm 5.70$ vs. $24.00 \pm 7.11 ; p=0.457)$ [Table 6]. The distribution of Majeed functional classification for group 1 was 8 excellent, 4 good, 2 fair, and 1 poor, whereas that for group 2 was 2 excellent, 4 good, 6 fair, and 5 poor [Table 7]. Thus group 1 demonstrated better functional results than group 2 at 1 -year follow up $(p=0.043)$.

There was no neurovascular complication in group 1, such as sciatic nerve palsy, but one case with superficial infection and debridement was required.

\section{DISCUSSION}

The most important finding in the present study was that patients treated with iliosacral screw fixation had improved functional outcomes compared with patients treated by conservative means. These results parallel the radiographic

Table 5: Results of short form health status survey (SF-36)

\begin{tabular}{lccc}
\hline & $\begin{array}{c}\text { Group 1 } \\
(n=15)\end{array}$ & $\begin{array}{c}\text { Group 2 } \\
(n=17)\end{array}$ & $p$ value \\
\cline { 2 - 3 } & \multicolumn{3}{c}{ Mean \pm SD } \\
\hline Physical functioning & $55.33 \pm 31.25$ & $50.88 \pm 22.92$ & 0.531 \\
Role-physical & $31.66 \pm 37.16$ & $17.64 \pm 21.22$ & 0.464 \\
Bodily pain & $51.93 \pm 26.01$ & $47.82 \pm 10.66$ & 0.235 \\
General health & $50.26 \pm 22.53$ & $34.64 \pm 15.71$ & $0.032^{*}$ \\
Vitality & $49.33 \pm 19.81$ & $42.35 \pm 10.62$ & 0.146 \\
Social functioning & $53.33 \pm 30.42$ & $50.73 \pm 12.86$ & 0.772 \\
Role-emotional & $60.00 \pm 47.47$ & $41.17 \pm 47.91$ & 0.263 \\
Mental health & $55.46 \pm 15.33$ & $48.23 \pm 7.93$ & $0.043^{*}$ \\
Physical component summary & $37.11 \pm 11.61$ & $34.22 \pm 6.74$ & 0.691 \\
Mental component summary & $42.91 \pm 10.13$ & $38.62 \pm 8.01$ & 0.166 \\
\hline Abbreviation: SD: Standard deviation; $* p<0.05$ & &
\end{tabular}

Table 6: Results of Majeed functional scores

\begin{tabular}{lccc}
\hline & $\begin{array}{c}\text { Group 1 } \\
(n=15)\end{array}$ & $\begin{array}{c}\text { Group 2 } \\
(n=17)\end{array}$ & $p$ value* \\
\cline { 2 - 3 } & \multicolumn{3}{c}{ Mean \pm SD } \\
\hline Pain & $25.00 \pm 7.32$ & $20.59 \pm 6.09$ & 0.028 \\
Work & $16.33 \pm 4.33$ & $10.57 \pm 4.60$ & $0.006^{* *}$ \\
Sitting & $8.00 \pm 1.85$ & $6.71 \pm 1.72$ & $0.049^{*}$ \\
Sexual intercourse & $3.00 \pm 1.00$ & $2.88 \pm 0.93$ & 0.473 \\
Standing & $26.27 \pm 5.70$ & $24.00 \pm 7.11$ & 0.457 \\
\hline
\end{tabular}

Abbreviation: SD: Standard deviation; $* p<0.05 ; * * p$ value $<0.01$

Table 7: Results of Majeed pelvis grade

\begin{tabular}{lccc}
\hline & Group 1 & Group 2 & $p$ value \\
\hline Excellent & 8 & 2 & $0.043^{*}$ \\
Good & 4 & 4 & \\
Fair & 2 & 6 & \\
Poor & 1 & 5 & \\
Total & 15 & 17 & \\
$* p<0.05$ & & &
\end{tabular}


results of less proximal vertical displacement in group 1 as compared with group 2 . The results are consistent with those of Papakostidis et al. ${ }^{[15]}$ whose systematic review of 1,641 patients demonstrated better radiological results for posterior pelvis internal fixation than for other therapeutic strategies. Minimizing the displacement of the hemi-pelvis seemed to be a reasonable approach, because less than $5 \mathrm{~mm}$ of residual displacement has been correlated with satisfactory results..$^{[16,17]}$ In the present study, group 1 had mean displacement of less than $5 \mathrm{~mm}$ along with better functional outcomes. Group 1 also had less pain in VAS assessment than group 2 at 1 -month $(p<0.001)$ and 1-year $(p=0.001)$ follow up. Moreover, percutaneous placement of iliosacral screws provided early and medium-term pain relief. On the other hand, poor outcomes were demonstrated in 2 patients in group 2 who underwent conservative treatment and had more than $10 \mathrm{~mm}$ of residual displacement. ${ }^{[18,19]}$ Early pelvic fracture stabilization has been suggested to facilitate early rehabilitation and reduce morbidity. ${ }^{[20]}$

The SF-36 is a standardized health status survey that provides a comprehensive, psychometrically sound, and efficient way to measure outcome from the patient's point of view. In the present study, functional outcomes assessment with the SF-36 showed better results in group 1 on the general health $(p=0.032)$ and mental health $(p=0.043)$ subscales at 1-year follow up. However, the broad scope of the SF-36 may preclude focusing on the specific problem of pelvic ring injury.

We also used the Majeed functional grading system, which allows easy and comprehensive assessment, including of specific problems caused by pelvic ring injury such as sitting or sexual intercourse. ${ }^{[14]}$ The Majeed functional scores were better for group 1 than for group 2 on the pain $(p=0.028)$, work $(p=0.006)$, and sitting $(p=0.049)$ subscales; moreover, group 1 had a higher proportion of patients with grades of excellent and good as compared with group 2. Although in previous studies sexual dysfunction was reported in $29 \%$ to $39 \%$ of patients after pelvic ring injuries ${ }^{[16,21]}$ no patients in the present study demonstrated sexual or excretory dysfunction because of the initial exclusion of patients with neurological or urological injuries. We used Majeed functional scores to evaluate discomfort with sexual intercourse, and more than half of patients $(68 \%)$ reported discomfort regardless of whether they received surgical treatment. We supposed that in most cases the discomfort was attributable to the pelvic injury. The purpose of the Majeed evaluation of sexual intercourse was to assess the severity level of discomfort or pain, rather than the presence or absence of dysfunction.

The Young-Burgess classification system allows classification of pelvic fractures according to whether they are stable (APC1, LC1) or unstable (APC2, APC3, LC2, LC3, VS, and combined-mechanism injury types). ${ }^{[22]}$ Long-term outcomes of conservatively treated injuries have been reported as acceptable in stable cases but not in unstable ones. ${ }^{[3]}$ For unstable fractures, achieving reduction and adequate stability can provide good results in terms of Majeed functional outcomes and reduction of low back pain. ${ }^{[3,17,23]}$ Internal fixation of the posterior element of the pelvic ring with iliosacral screws through a posterior approach was suggested as an alternative for treating unstable pelvic fractures. ${ }^{[24-26]}$ Percutaneous screw placement indeed minimized violation of compromised soft tissue and reduced intraoperative blood loss as well as the rate of postoperative infection. ${ }^{[6]}$

Limitations of the present study must be acknowledged. First, the study sample size was small because isolated pelvic posterior ring injury is uncommon. Nevertheless, statistically significant differences were demonstrated. As this is a retrospective and non-randomized study, some confounding bias may lead to differences in the characteristics between the groups. Second, long-term functional results were not addressed and warrant further study. Finally, although iliosacral screw fixation under fluoroscopic guidance has been reported to be safe and effective, the limited target zone near the neuroforamen may still carry a risk of nerve root injury. Navigation systems based on intraoperative computed tomography may allow more comprehensive and accurate imaging for screw placement.

In conclusion, percutaneous placement of iliosacral screws for the treatment of unstable posterior pelvic ring injuries can result in less residual displacement, better pain relief, and better functional outcomes at 1-year follow up than conservative treatment methods.

\section{REFERENCES}

1. van Gulik TM, Raaymakers EL, Broekhuizen AH, Karthaus AJ. Complications and late therapeutic results of conservatively managed, unstable pelvic ring disruptions. Neth J Surg 1987;39:175-8.

2. Miranda MA, Riemer BL, Butterfield SL, Burke CJ $3^{\text {rd }}$. Pelvic ring injuries: A long term functional outcome study. Clin Orthop Relat Res 1996;329:152-9.

3. Henderson RC. The long-term results of nonoperatively treated major pelvic disruptions. J Orthop Trauma 1989;3:41-7.

4. Matta JM, Tornetta $\mathrm{P} 3^{\text {rd }}$. Internal fixation of unstable pelvic ring injuries. Clin Orthop Relat Res 1996;329:129-40.

5. Sagi HC, Militano U, Caron T, Lindvall E. A comprehensive analysis with minimum 1-year follow up of vertically unstable transforaminal sacral fractures treated with triangular osteosynthesis. J Orthop Trauma 2009;23:313-9; discussion 319-21.

6. Shuler TE, Boone DC, Gruen GS, Peitzman AB. Percutaneous iliosacral screw fixation: Early treatment for unstable posterior pelvic ring disruptions. J Trauma 1995;38:453-8.

7. Tornetta P $3^{\text {rd }}$, Matta JM. Outcome of operatively treated unstable posterior pelvic ring disruptions. Clin Orthop Relat Res 1996;329:186-93. 
8. Routt ML Jr, Kregor PJ, Simonian PT, Mayo KA. Early results of percutaneous iliosacral screws placed with the patient in the supine position. J Orthop Trauma 1995;9:207-14.

9. Routt ML Jr., Simonian PT, Agnew SG, Mann FA. Radiographic recognition of the sacral alar slope for optimal placement of iliosacral screws: A cadaveric and clinical study. J Orthop Trauma 1996;10:171-7.

10. Keating JF, Werier J, Blachut P, Broekhuyse H, Meek RN, O'Brien PJ. Early fixation of the vertically unstable pelvis: The role of iliosacral screw fixation of the posterior lesion. J OrthopTrauma 1999;13:107-13.

11. Routt ML Jr, Kregor PJ, Simonian PT, Mayo KA. Early results of percutaneous iliosacral screws placed with the patient in the supine position. J Orthop Trauma 1995;9:207-14.

12. DonTigny RL. Function and pathomechanics of the sacroiliac joint: A review. Phys Ther 1985;65:35-44

13. Burgess AR, Eastridge BJ, Young JW, Ellison TS, Ellison PS Jr, Poka A, et al. Pelvic ring disruptions: Effective classification system and treatment protocols. J Trauma 1990;30:848-56.

14. Majeed SA. Grading the outcome of pelvic fractures. J Bone Joint Surg Br 1989;71:304-6.

15. Papakostidis C, Kanakaris NK, Kontakis G, Giannoudis PV. Pelvic ring disruptions: Treatment modalities and analysis of outcomes. Int Orthop 2009;33:329-38.

16. Cole JD, Blum DA, Ansel LJ. Outcome after fixation of unstable posterior pelvic ring injuries. Clin Orthop Relat Res 1996;329:160-79.

17. Lindahl J, Hirvensalo E. Outcome of operatively treated type-C injuries of the pelvic ring. Acta Orthop 2005;76:667-78.
18. Lindahl J, Hirvensalo E, Böstman O, Santavirta S. Failure of reduction with an external fixator in the management of injuries of the pelvic ring: Long-term evaluation of 110 patients. J Bone Joint Surg Br 1999;81:955-62.

19. McLaren AC, Rorabeck CH, Halpenny J. Long-term pain and disability in relation to residual deformity after displaced pelvic ring fractures. Can J Surg 1990;33:492-4.

20. Latenser BA, Gentilello LM, Tarver AA, Thalgott JS, Batdorf JW. Improved outcome with early fixation of skeletally unstable pelvic fractures. J Trauma 1991;31:28-31.

21. Tötterman A, Glott T, Søberg HL, Madsen JE, Røise O. Pelvic trauma with displaced sacral fractures: Functional outcome at one year. Spine (Phila Pa 1976) 2007;32:1437-43.

22. Manson T, O'Toole RV, Whitney A, Duggan B, Sciadini M, Nascone J. Young-Burgess classification of pelvic ring fractures: Does it predict mortality, transfusion requirements, and non-orthopaedic injuries? J Orthop Trauma 2010;24:603-9.

23. Sen RK, Veerappa LA. Outcome analysis of pelvic ring fractures. Indian J Orthop 2010;44:79-83

24. Comstock CP, van der Meulen MC, Goodman SB. Biomechanical comparison of posterior internal fixation techniques for unstable pelvic fractures. J Orthop Trauma 1996;10:517-22.

25. Gunterberg B, Goldie I, Slätis P. Fixation of pelvic fractures and dislocations: An experimental study on the loading of pelvic fractures and sacro-iliac dislocations after external compression fixation. Acta Orthop Scand 1978;49:278-86.

26. Letournel E. Pelvic fractures. Injury 1978;10:145-8. 